\title{
HEALS: A Faith-Based Hypertension Control and Prevention Program for African American Churches: Training of Church Leaders as Program Interventionists
}

\author{
Sunita Dodani, ${ }^{1}$ Debra Sullivan, ${ }^{2}$ Sydney Pankey, ${ }^{3}$ and Catherine Champagne ${ }^{4}$ \\ ${ }^{1}$ Center for Post Polio Rehabilitation (A Nonprofit Organization), 2308 W, 127 Street, Leawood, KS 66209, USA \\ ${ }^{2}$ Department of Nutrition, University of Kansas Medical Center, Kansas City, KS 66103, USA \\ ${ }^{3}$ Department of Preventive Medicine, University of Kansas Medical Center, Kansas City, KS 66103, USA \\ ${ }^{4}$ Pennington BioMedical Research Center, Baton Rouge, LA 70808-4124, USA \\ Correspondence should be addressed to Sunita Dodani, soni.dodani@gmail.com
}

Received 27 February 2011; Accepted 17 March 2011

Academic Editor: Samy I. McFarlane

Copyright () 2011 Sunita Dodani et al. This is an open access article distributed under the Creative Commons Attribution License, which permits unrestricted use, distribution, and reproduction in any medium, provided the original work is properly cited.

\begin{abstract}
Background. A 12-session church-based HEALS program (healthy eating and living spiritually) was developed for hypertension control and prevention program in African Americans (AAs). This study presents specifics of training lay health educators to effectively deliver HEALS to high-risk AAs. Methods. A one-day workshop was conducted by the research experts in an AA church. Five church members were recruited to be program interventionists called church health counselors (CHCs). Results. Using principles of adult education, a training protocol was developed with the intention of recognizing and supporting CHCs skills. $\mathrm{CHCs}$ received training on delivering HEALS program. The process of training emphasized action methods including role playing and hands-on experience with diet portion measurements. Conclusion. With adequate training, the community lay health educator can be an essential partner in a community-based hypertension control programs. This may motivate program participants more and encourages the individual to make the behavior modifications on a permanent basis.
\end{abstract}

\section{Introduction}

Hypertension (known as the "silent killer") prevalence is highly variable among populations worldwide. In the US, there is a disproportionate burden of hypertension and its complications in African Americans (AAs) [1]. Not only are AAs more likely to suffer from hypertension than are whites, but they also experience a higher complication rate, greater severity, and earlier disease onset. From 1988-1994 to 19992002 , the prevalence of hypertension in adults increased from $35.8 \%$ to $41.4 \%$ among AAs, and it was particularly high among AA women, at $44.0 \%$ compared to $28.1 \%$ in whites [2]. Hypertension is more common in middle-aged or older, less-educated, overweight or obese, and physically inactive AAs [3]. As a result, compared with whites, AAs have a 1.3times greater rate of nonfatal stroke, a 1.8-times greater rate of fatal stroke, a 1.5-times greater rate of heart disease death, and a 4.2-times greater rate of end-stage kidney disease $[4,5]$. Many AAs, however, remain unaware of their blood pressure status [5].
It is common, asymptomatic, readily detectable, and easily treatable. Yet it burdens our economy substantially. Hypertension is the most common primary diagnosis in the US and accounts for over 38 million office visits per year [6]. Most patients will require two or more antihypertensive medications to achieve the goal of blood pressure less than $140 / 90$ or $130 / 80$ for patients with diabetes or kidney disease $[7,8]$. Poor adherence to medication regimens is a common problem with AAs who have hypertension, perhaps because it is often asymptomatic in the early stages [9]. A high-cost prescription can further decrease adherence to antihypertensive medications. Therefore, emphasis on disease prevention and control are the primary means by which we can begin to reign in unnecessary medical expenditures and promote a healthier society.

Besides medications, lifestyle interventions are also effective in lowering blood pressure (BP), and the "dietary approach to stop hypertension" (DASH) diet has been shown to lower systolic BP (SBP) by $6-14 \mathrm{mmHg}$, equivalent to one antihypertensive agents [10]. However, in the PREMIER 
trial, DASH with lifestyle modifications was less effective in AAs with regard to BP lowering effects [11].

It has been shown that promoting the adoption of healthy lifestyles, ones that involve best practices of diet and exercise and abundant expert support, can, in a healthcare setting, reduce the incidence of hypertension in those who are at high risk and as well to its control [11]. Using faithbased settings and adopting community-based participatory research (CBPR) approach, especially for AAs, hypertension control programs can be developed and offered by faithcommunities. For a number of years, there has been a trend to have lay health leaders rather than health professional to deliver health promotion and disease prevention programs to hard-to-reach community [12]. Normally, these lay educators are residents of the same communities in which they conduct the program. There are several strengths to using lay health educators/advisors (supported by experts and church pastors) in delivering CBPR programs: (i) they are acceptable to hard-to-reach community residents, (ii) they provide a ready support network, (iii) they serve as efficacious peer models, (iv) they promote community ownership, and (v) they are cost-effective [12]. Many members of AAs churches may have significant experience dealing with people who have hypertension as patients or as caregivers to relatives and friends. The potential for developing church health counselors (CHCs) who devote their time to hypertension prevention and management remains virtually untapped and is a potential future offshoot of this effort. The idea of having the church health leaders educate and support at-risk and healthy individuals and not just pray for the sick is consistent with the $\mathrm{CHC}$ model.

We have developed HEALS (healthy eating and living spiritually), a hypertension control and prevention program with the help of the AA community, CBPR experts and community advisory board (CAB) input. HEALS has been modified from the DASH [10] and PREMIER [11] studies. Results of focus groups and program development will be published elsewhere. HEALS program, a church-based approach, if successful, may be utilized to reduce the incidence and consequences of hypertension in large communities with potentially huge impact on public health by implementation in community settings such as church venues.

The sizeable AA communities in the Kansas City Metro area (Kansas and Missouri) make it an ideal population in which to implement a hypertension control and prevention program. For this reason, we have proposed the implementation of a faith-based hypertension control and prevention program be piloted in a small Kansas City church.

Lay health educators have been used successfully in a wide range of community settings and with a variety of targeted health issues. For example, lay health educators were used in Fit Body and Soul study [12], Project Joy [13], PATHWAYS [14], Pawtucket Heart Health Program [15], Stanford Five City Project [16], and many more. The proposed HEALS is a multilevel CBPR intervention for hypertension control and management of high risk AAs in churches. The program started in different phases $[13,14]$. An abundance of literature exists on the outcome of community based health promotions programs that use community lay educators as interventionists. However, very little has been published on the specifics of training lay health educators to effectively deliver community-based programs. The objective of current study is to share the experience of training church lay health educators as HEALS interventionists who will deliver a 12session program under the supervision of the research team and DASH and PREMIER experts.

\section{Materials and Methods}

This was a one-day workshop conducted by the research team members and experts of DASH and PREMIER studies at the Allen Chapel A.M.E. church in Kansas City, Kansas. For this pilot study, the 200 member Allen Chapel A.M.E. was selected for several reasons (i) members having lower or middle lower socioeconomic and demographic characteristics, (ii) a high level of interest in the project, (iii) existence of a health awareness programs within the church, (iv) Church is part of university community advisory board (CAB), and (v) PI's existing relationship with the church pastor. The church pastor was asked to nominate at least 4-5 health ministers who are at least 25 years old, preferably belonging to the health profession, have effective communication skills, and are willing to make a 6 month commitment to the study. There were no requirements for the prior training in health education or in health care, but the importance of church-based lay health educators being viewed as role models among program participants was emphasized. Five church members were recruited and were called church health counselors (CHCs). All five CHCs (four females and one male) are usually known "influence leaders" who were highly respected in the church and often included in the major church decisions. CHCs ranged in age from 35 to 63 years (average age $=50$ years) one of the $\mathrm{CHCs}$ had nursing experience however, none had any formal training or experience in conducting small group faith-based lifestyle program. All five received the HEALS program training (described below) and are part of the church advisory panel. Because of their time commitment, CHCs are provided with a small stipend to offset their out-of-pocket expenses and to serve as an incentive for participating in the study. The focus group meetings were held prior to the start of the project. The church pastor and five CHCs with the PI discussed the project concept, set an outline of activities, and marked dates for all activities. Because of the pilot nature of this study, power analysis was not conducted and a convenience sample of 20-25 overweight or obese church members who are either high risk for hypertension (with high normal and stage 1 hypertension) and/or are known hypertensive's will be enrolled in the study after screening. For this study, all participants are members of Allen Chapel A.M.E. church.

Before describing the experiences of training $\mathrm{CHCs}$, we briefly discuss here the community advisory board (CAB).

\section{Health Disparity Community Advisory Board (CAB)}

The Kansas University Medical Center (KUMC) under the leadership of the Dr. Dodani established a Health Disparities 
Community Advisory Board (CAB), funded by the KUMCDepartment of Internal Medicine and Center for Outcome Research and Education (CORE)—http://www2.kumc .edu/internalmedicine/.

The 45 member $\mathrm{CAB}$ has more than $70 \%$ representation of AA communities. In addition, $\mathrm{CAB}$ also includes (i) other ethnic minority representation, (ii) local public/private/government organizations serving low-income ethnic minority groups, (iii) churches, and (iv) non-profit organizations. The primary functions of the $\mathrm{CAB}$ are to (1) identify health issues from the perspective of community members, (2) promote communication among community leaders and researchers, (3) promote partnership and ownership by community members in research projects that involve, and have an impact on, the overall health of the AA community, and (4) bring together community leaders and researchers to design, implement, and evaluate culturally sensitive, practical research projects. The $\mathrm{CAB}$ already provided significant input and oversight towards the development of the current application. In addition, $\mathrm{CAB}$ (majority AA members) participated in the HEALS program development and provided appropriate bible scripture for the program sessions [12]. Indeed, the $C A B$ perceives the faith-based settings as having the strongest and most credible influences among AAs in the community. During the proposed study, the $\mathrm{CAB}$ will continue to meet on average 3-4 times per year and play a critical role in promoting mutual understanding and cooperation between researchers and participant community members. The $\mathrm{CAB}$ will also strongly encourage and develop long-term commitments to various health-promoting activities that will be selfsustained by the participants in the study, thereby improving the overall health of the AA community far beyond those achieved by merely participating in the study. In this regard, in close collaboration with the researchers, the $C A B$ will develop various potential strategies of sustainable life-style modifications that can be applied to this unique setting of AA community.

\section{Development of 12-Session HEALS Program}

Briefly, the HEALS is a small-group, behavior-oriented hypertension control and prevention program targeted for high risk AA church members who are either at the risk of future hypertension (those with high normal blood pressure or stage I hypertension) for those who are known hypertensives. HEALS is a faith-based hypertension control and prevention intervention, developed using CBPR approaches and input from members of AAs churches; it was developed during a multistep process and was approved by the University of Kansas Medical Center institutional review board. The basis of the HEALS was DASH (a feeding study-clinical trial) and PREMIER (an 18-lifestyle-month) intervention which focused on the DASH dietary pattern [11]. Sessions of PREMIER were modified with modification of DASH diet to make a culturally appropriate diet plan without compromising the integrity of DASH caloric contents. Faithbased strategies of Fit Body and Soul [12] were used to add faith components in the multilevel HEALS church-based program. Members of the original DASH and PREMIER lifestyle team and $C A B$ active members from more than 10 churches were involved in the modification and development of the program. This group of church leaders and experts modified the PREMIER sessions, using the experiences of the Fit Body and Soul study [12] and input from the CAB advisory panel. They developed the 12-session faith-and culture-based program for the participants in the study (the full program can be provided upon request). The HEALS program was formed within a spiritual framework. Working with the advisory board of church leaders, we facilitated the development of the content and design of the manual. Board members selected the spiritual themes and scripture to frame the 3 themes of the intervention-weight loss, increased PA, behavioral change-and wrote messages to be included in the manual. The spirituality was seen as a source of emotional support, a positive influence on health, and contributing to life satisfaction. Overall feedback from the advisory panel and 3-4 focus groups regarding this multilevel, spirituality-based, behavioral lifestyle intervention for AA congregations was generally consistent, showing strong preferences for design and content. In order to achieve outcomes and goals (Table 1), the multi-level spiritually based HEALS behavioral lifestyle intervention was designed to be delivered using three different approaches: church level (led by pastor); group level (led by CHCs), and individual level approach (led by $\mathrm{CHCs}$ ).

\section{Results and Discussion}

5.1. CHCs Training Workshop on HEALS Program. The purpose of this training workshop was to familiarize CHSs with all HEALS material as well as appropriate intervention measures. The model for developing and conducting training was based on the principles of adult education [17]. Adult learners bring to training a wealth of experience and welldeveloped skills. As such, the training of adult learners should reinforce and expand upon the learner's prior experience and direct existing skills to new applications. This process of adult learning is facilitated by instruction that is goal oriented and personally relevant to the learner one that actively involves the learner in role playing and other learning exercises and that affords frequent feedback. A blend of lecture, hands-on practice, in person demonstrations, and other methodologies consistent with adult learning theories were employed as teaching techniques. CHCs received the program manual guides, checklist protocols, and the rationale behind these approaches. These key features of adult learning were emphasized in the training program.

The learning objectives of the workshop were to

(1) understand the importance of the HEALS program and its intended outcome,

(2) have knowledge of healthy meals and how to prepare them with understanding of calory counts,

(3) understand the importance of proper documentation and completion of instruments for diet recalls, physical activity, and other questionnaires, 
TABLE 1: HEALS Goals at 3 levels: (1) Church, (2) Group, (3) Individual.

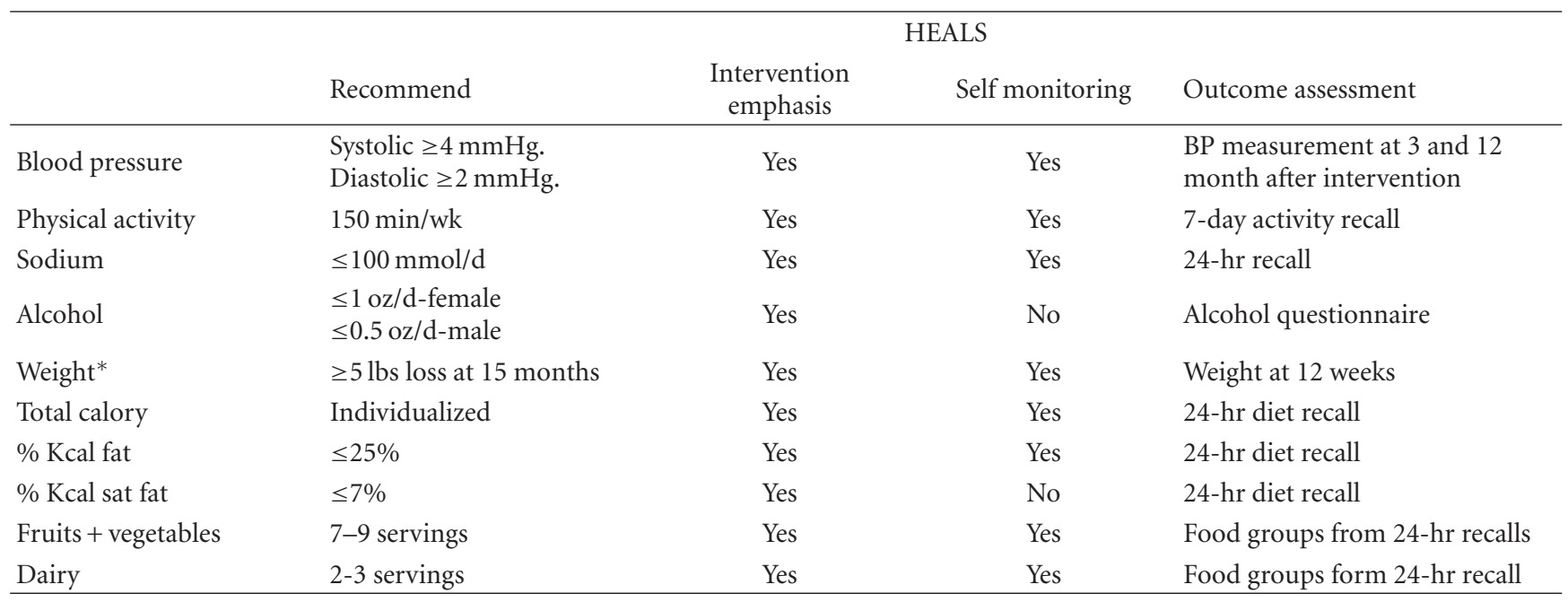

*Weight loss recommended only for those with $\mathrm{BMI} \geq 25$, daily number of servings adjusted for individual caloric intake.

(4) know how to take blood pressure, height, weight, pedometer readings, waist circumference, and BMI measurements and record in participants' data forms,

(5) maintain log dairies,

(6) understand barriers to and provide acceptable solutions to study participants on maintaining a healthy lifestyle.

This one-day workshop was conducted at the Allen Chapel A.M.E. church in the Kansas City, Kansas. Within the church, all 5 CHCs were provided a one-time (approx $8 \mathrm{hrs}$ ) formal training. Training sessions were conducted by research experts including a PREMIER and DASH investigators with background in behavioral education, psychology, nutrition, and faith-based CBPR programs (Figure 1). The training protocol was developed with the intention of recognizing and supporting our CHCs existing skills and experiences. The process of training emphasized action methods including simulation, role playing, and hands-on experience with diet portion measurements.

In the first session (by study PI), the project overview, timelines, CHCs responsibilities and the roles of research team were clarified. The clarification of roles and responsibilities served to establish a collaborative partnership and positioning university project personnel in a supportive role while affording each $\mathrm{CHC}$ the opportunity with autonomy and at the same time be supervised by the research team within the parameters of study protocol. In these negotiation roles, each $\mathrm{CHC}$ had a significant input on a number of issues including program session timings, program scheduling, design of participant recruitment activities, and materials. The first session also included the overview of the philosophy of HEALS program, the importance of faith-based approach, behavioral goal setting, and the importance of multilevel approach: church, group, and individual level. All CHCs were provided with the copies of HEALS manual which included learning objectives of each session, instructors scripts, and participants materials for all 12 sessions.
After reviewing the overall objectives and goals of HEALS program, the PREMIER and DASH team investigator presented the overall objectives of each of the 12 sessions with emphasis on the approach, method of delivery and outcome measures. A majority of the emphasis was made on the achievement of healthy behaviors and how to maintain them. CHCs discussed some of eating habits in the church settings and provided examples how those habits could be modified into healthy eating. Enough time was provided to $\mathrm{CHCs}$ to make sure they understood the basics of each session and received clarification. $\mathrm{CHCs}$ also brainstormed on strategies for recruiting program participants from among their church congregation and neighboring communities. CHCs then practiced conducting HEALS session and received feedback, guidance and tips from the research team members.

The third session was conducted by the research team nutritionists (study coinvestigator) who introduced $\mathrm{CHCs}$ to different types of foods, food servings, calory content measurement method, measurement of portion sizes, and the importance of portion of fat, carbohydrates, proteins, and vitamins in making meal menus (Figure 2). The CHCs also performed some hands-on exercises by role playing. The use of role playing method in the training provided them with the type of challenging questions and group scenarios that they would likely encounter, based on the prior program experience of the research team. These included easy methods of measuring portion sizes, how to read and understand food labels, knowledge of alternative foods to reduce fat intake, helping participants set reasonable goals, and promoting self-efficacy for behavior change. The handson exercises also encouraged team building among the $\mathrm{CHCs}$ through shared session preparation and planning and allowed for self-evaluation and constructive feedback from their fellow CHCs and research team.

Finally, CHCs were briefed about methods and protocols of anthropometric measurements. All CHCs also received refresher training before the screening kickoff event and $\mathrm{CHCs}$ practiced taking anthropometric measurements on 


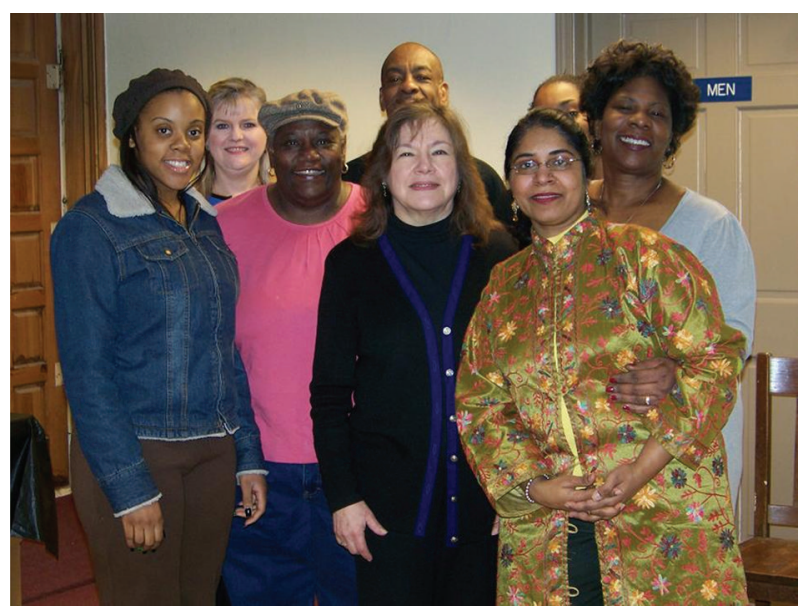

Figure 1: HEALS Team with CHCs (from left to right front row). Alexis Belcher (CHC), Ivey Brown (CHC) Catherine Champagne (study Co-I), and Sunita Dodani Debra Sullivan (study PI). Back row: Debra Sullivan (study Co-I), Thaerius Berry (CHC), Sydny Pankey (study coordinator), and Vera Brown (CHC).

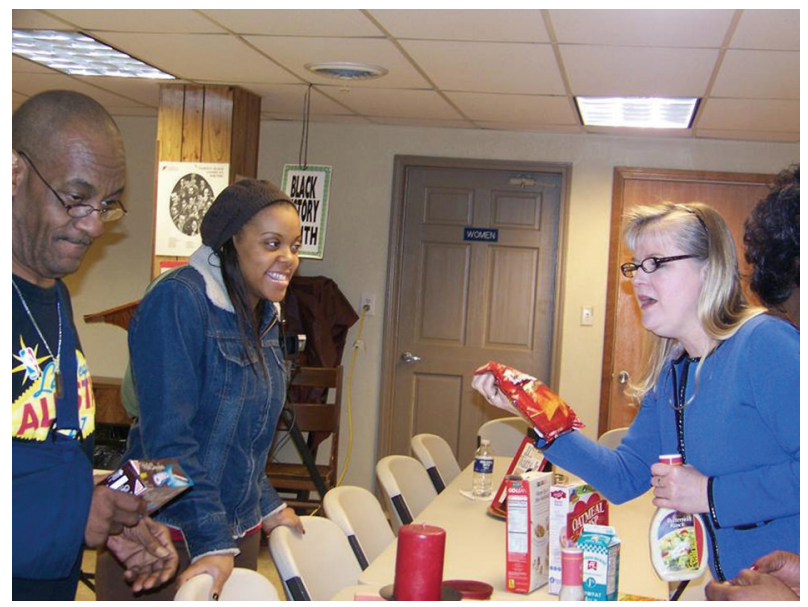

FIGURE 2: Demonstration of food servings and calory content measurement method.

each other, as well as on research team members. In addition, $\mathrm{CHCs}$ continued role playing and reviewing HEALS sessions in order to have a full understanding of the program. CHCs received feedback from the research team designed in such a way that it was both collaborative and constructive while allaying any potential for embarrassment and defensiveness.

In addition to conducting program sessions under the supervision of experts, CHCs were asked to be responsible for a number of activities involved in operating the program therefore, clear demonstration and education related to other activities were also included in the training. These responsibilities included, but were not limited to (a) publicity and awareness of the program within the church, (b) participants' recruitment from the church, (c) scheduling sessions and notifying participants of meetings, (d) planning, organizing and conducting the 12 program sessions, (e) making weekly calls to participants for followup, diary check, and providing solutions to any barriers towards achieving program goals, and (f) maintaining follow-up contacts with any participants who missed session/s. The research team served as the source of information and advice to the CHCs throughout the program activities. More practice time and skill testing with standardized checklist protocols were incorporated in other church/program-related activities, for example, screening and participant recruitment events.

5.2. Discussion. With cardiovascular disease the leading cause of death and disability in the US, economically feasible and effective methods of prevention are needed. Despite more than 30 years of intense activity to improve control and more recently prevention, high blood pressure (hypertension) continues to be a major public health problem. Evidence-based reviews have identified best practices and quality-improvement strategies to address prevention and control [5]. Since the 1970s, community-based programs have been instrumental in raising awareness, increasing knowledge, and promoting changes in health behavior to improve blood pressure control. Most of these programs have emphasized the use of partnerships and involvement of community residents in conducting screening and referral activities, implementing clinical practice guidelines, and increasing healthy eating and physical activity. Many have also used health care team approaches, including the use of trained community health workers to deliver targeted, culturally sensitive health education, particularly related to the prevention of cardiovascular disease risk factors in general and hypertension in particular.

Health education programs that target hard-to-reach communities are often delivered by lay community persons who are trained to serve as lay health educators. While these community lay health educators bring a number of strengths to CBPR programs, the effectiveness of these volunteerdelivered programs in community and church settings is to large extent dependent upon the training afforded to the lay health educators. Without the proper training and practice, the delivery of the program and anticipated outcomes may be unnecessarily compromised. The amount and type of training required for the church-based lay health educators to reliably and effectively implement a faith-based hypertension control and prevention program are likely to be affected by several factors. Some of the important factors are (a) the complexity of the targeted health behaviors, (b) full understanding of the program participants and community, (c) the specialized knowledge and skills required to implement the program, and (d) relative independence of the community health educators to implement the program. Unfortunately, the literature on lay health educator's training often fails to discuss the specifics of training and/or importance of training. This paper was an attempt to shed light on specific components of training HEALS program lay health educators called CHCs (church health counselors). This paper also describes the training used to prepare church-based CHCs to conduct HEALS hypertension control and prevention healthy lifestyle program, modified from DASH and PREMIER studies to 
make it suitable for AA faith communities. These $\mathrm{CHCs}$ helped the team in arranging screening events at the church as well as subsequent recruitment of eligible participants that will receive the 12 -session HEALS from these CHCs under the supervision of team experts.

The training needs of lay health educators, particularly in this study, are heavily influenced by the degree of structure inherent in the program that they will deliver. The HEALS is a highly structured program that includes carefully designed scripts, input from the faith-based community leaders, easy understandable session goals for CHCs to deliver, and selfdirected learning activities for participants. In this study, the training provided to the church $\mathrm{CHCs}$ made no attempt to foster an in-depth knowledge of PREMIER or DASH studies, or understanding of nutrition or dietary modification principles, although the study nutritionist was available as a resource to the church $\mathrm{CHCs}$ and participants. The objective of the training was the focus on the delivery of program information and activities included in the structured HEALS program. The training provided to lay health educators emphasized the common components of each sessions, including bible scriptures selected by the church and $C A B$ appropriately to suit the main theme of each session. The complete HEALS program manual (instructor's manual) provided the detailed scripts for the delivery of each session by respective CHCs. The results of this study highlight the importance of training of lay health educators who will be responsible for the delivery of behavioral lifestyle program and hence the expected outcomes. We believe that even a complex multilevel lifestyle faith-based program, like HEALS, can be effectively administered by lay church members as long as program is sufficiently structured and adequate training and support are provided.

\section{Conclusion}

Hypertension (and hence high blood pressure) is a significant health problem affecting AAs. Community outreach programs in general and faith-based programs in particular can be valuable in reaching at-risk groups. Community lay health educators can greatly facilitate both access to hardto-reach community and the implementation of programs designed for high-risk community/church members. In training lay educators to implement church-based behavioral lifestyle programs, it is not essential to create content experts. With adequate training, the community lay health educator can be an essential partner in a community-based lifestyle hypertension control and prevention program. This in, return, may motivate program participants more and encourages the individual to make the behavior modifications on a permanent basis, when program is presented by their own church people.

\section{Financial Disclosures}

The authors have nothing to disclose.

\section{Conflict of Interests}

The authors declare there is no conflict of interests.

\section{Ethics Approval}

The study was conducted with the understanding and the consent of the human subject. This study was conducted with the approval of the University of Kansas Medical Center ethics board.

\section{Acknowledgments}

The authors are deeply appreciative of Rev Stacy Evans, pastor of Allen Chapel A.M.E. church who supported Dodani and partnered with the research team to conduct this pilot project at the church. They also want to thank all church health counselors (Alexis Belcher, Thaerius Berry, Ivey Brown, Thelma Poole, and Vera Watson) for their time, commitment, and interest in the program and acceptance to be the program leaders. They thank the University of Kansas Medical Center, Department of Internal medicine, for their full support with the HEALS project.

\section{References}

[1] W. Rosamond, K. Flegal, G. Friday et al., "Heart disease and stroke statistics-2007 Update: a report from the American Heart Association Statistics Committee and Stroke Statistics Subcommittee," Circulation, vol. 115, no. 5, pp. e69-e171, 2007.

[2] R. P. Hertz, A. N. Unger, J. A. Cornell, and E. Saunders, "Racial disparities in hypertension prevalence, awareness, and management," Archives of Internal Medicine, vol. 165, no. 18, pp. 2098-2104, 2005.

[3] A. V. Chobanian, G. L. Bakris, H. R. Black et al., "Seventh report of the joint national committee on prevention, detection, evaluation, and treatment of high blood pressure," Hypertension, vol. 42, pp. 1206-1252, 2003.

[4] C. D. Ford, M. J. Kim, and B. L. Dancy, "Perceptions of hypertension and contributing personal and environmental factors among rural southern African American women," Ethnicity and Disease, vol. 19, no. 4, pp. 407-413, 2009.

[5] L. S. Hicks, D. G. Fairchild, M. S. Horng, E. J. Orav, D. W. Bates, and J. Z. Ayanian, "Determinants of JNC VI guideline adherence, intensity of drug therapy, and blood pressure control by race and ethnicity," Hypertension, vol. 44, no. 4, pp. 429-434, 2004.

[6] National Center for Health Statistics, "Hypertension," 2006, http://www.cdc.gov/nchs/fastats/hyprtens.htm.

[7] H. R. Black, W. J. Elliott, G. Grandits, P. Grambsch, J. D. Neaton, and P. Sleight, "Predictors of blood pressure control in the CONVINCE trial," American Journal of Hypertension, vol. 17, p. 178A, 2004.

[8] W. C. Cushman, C. E. Ford, J. A. Cutler et al., "Success and predictors of blood pressure control in diverse North American settings: the antihypertensive and lipid-lowering treatment to prevent heart attact trial (ALLHAT)," Journal of Clinical Hypertension, vol. 4, no. 6, pp. 393-404, 2002. 
[9] M. Burnier, "Medication adherence and persistence as the Cornerstone of effective antihypertensive therapy," American Journal of Hypertension, vol. 19, no. 11, pp. 1190-1196, 2006.

[10] L. J. Appel, T. J. Moore, E. Obarzanek et al., "A clinical trial of the effects of dietary patterns on blood pressure," New England Journal of Medicine, vol. 336, no. 16, pp. 1117-1124, 1997.

[11] L. J. Appel, "Effects of comprehensive lifestyle modification on blood pressure control: main results of the PREMIER clinical trial," Journal of the American Medical Association, vol. 289, no. 16, pp. 2083-2093, 2003.

[12] S. Dodani and J. Z. Fields, "Implementation of the fit body and soul, a church-based life style program for diabetes prevention in high-risk African Americans: a feasibility study," Diabetes Educator, vol. 36, no. 3, pp. 465-472, 2010.

[13] L. R. Yanek, D. M. Becker, T. F. Moy, J. Gittelsohn, and D. M. Koffman, "Project joy: faith based cardiovascular health promotion for African American women," Public Health Reports, vol. 116, no. 1, pp. 68-81, 2001.

[14] W. McNabb, M. Quinn, J. Kerver, S. Cook, and T. Karrison, "The PATHWAYS church-based weight loss program for urban African-American women at risk for diabetes," Diabetes Care, vol. 20, no. 10, pp. 1518-1523, 1997.

[15] C. B. Eaton, K. L. Lapane, C. E. Garber, K. M. Gans, T. M. Lasater, and R. A. Carleton, "Effects of a community-based intervention on physical activity: the Pawtuckett Heart Health Program," American Journal of Public Health, vol. 89, no. 11, pp. 1741-1744, 1999.

[16] S. P. Fortmann and A. N. Varady, "Effects of a communitywide health education program on cardiovascular disease morbidity and mortality: the stanford five-city project," American Journal of Epidemiology, vol. 152, no. 4, pp. 316-323, 2000.

[17] A. B. Knox, Helping Adults Learn. A Guide to Planning, Implementing and Conducting Program, Jossey-Bass, San Francisco, Calif, USA, 1986. 


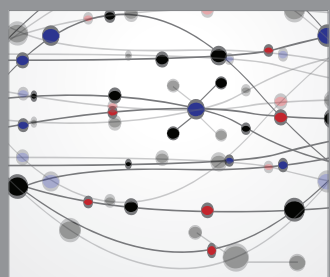

The Scientific World Journal
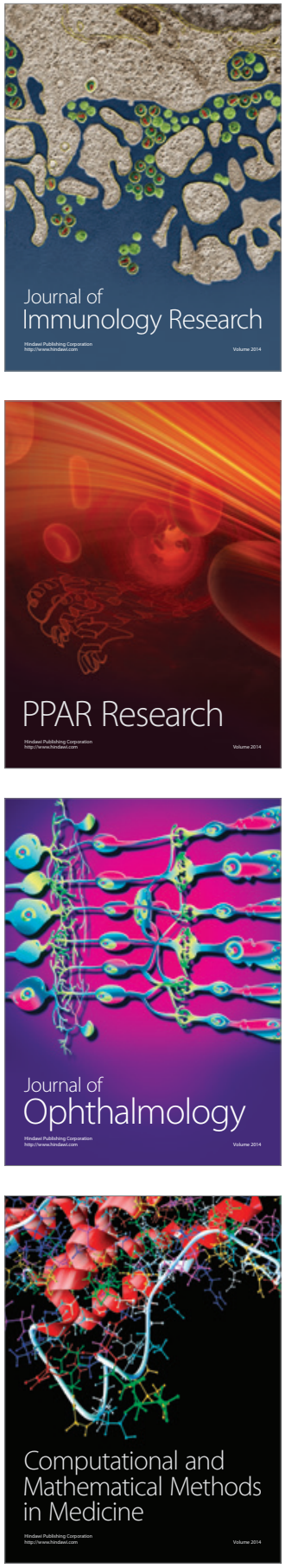

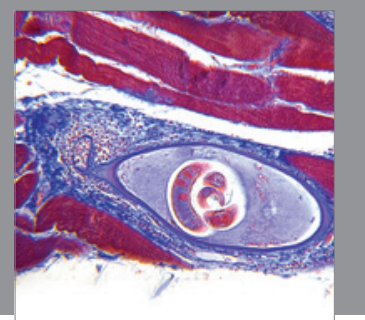

Gastroenterology

Research and Practice
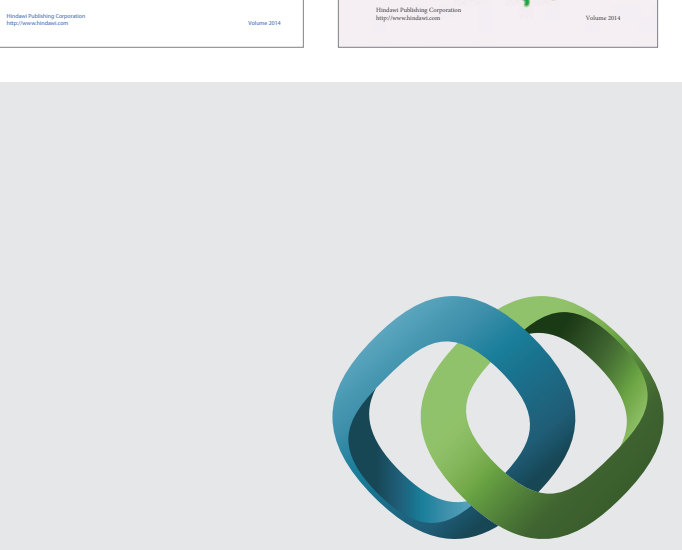

\section{Hindawi}

Submit your manuscripts at

http://www.hindawi.com
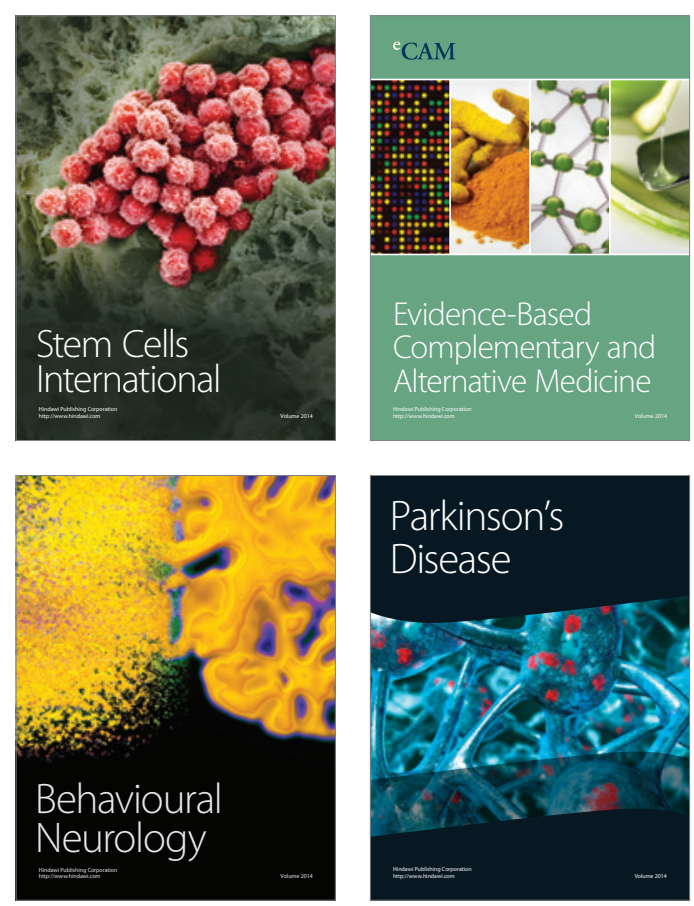

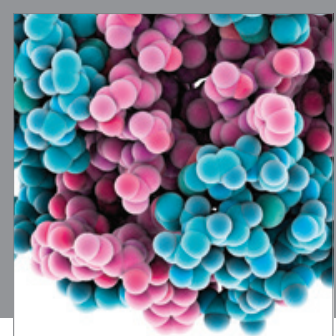

Journal of
Diabetes Research

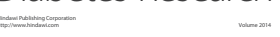

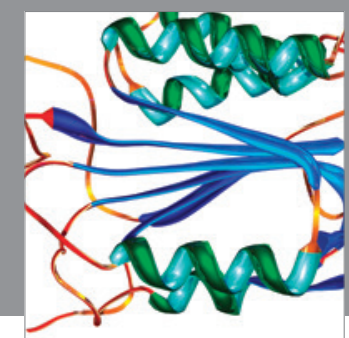

Disease Markers
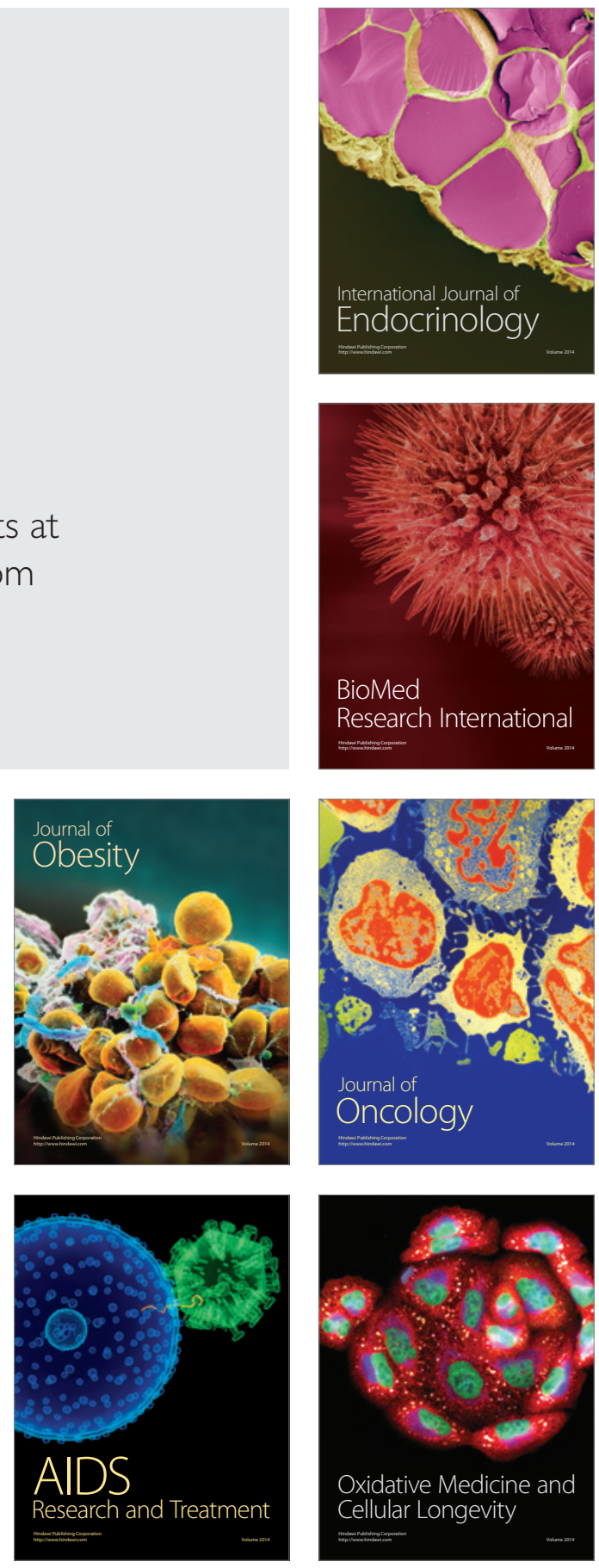